\title{
A Case Report on Gallstone Ileus Treated with the Endoscopy
}

\section{Masnobu Kishimoto, Yasutaka Okamoto, Takashi Muroya, Kentaro Kajino, Hitoshi Ikegawa, Yasuyuki Kuwagata}

Department of Emergency and Critical Care Medicine, Kansai Medical University, Hirakata, Japan

Email: kishimom@hirakata.kmu.ac.jp

How to cite this paper: Kishimoto, M., Okamoto, Y., Muroya, T., Kajino, K., Ikegawa, H. and Kuwagata, Y. (2021) A Case Report on Gallstone Ileus Treated with the Endoscopy. Open Journal of Emergency Medicine, 9, 188-195.

https://doi.org/10.4236/ojem.2021.94019

Received: October 25, 2021

Accepted: December 7, 2021

Published: December 10, 2021

Copyright $\odot 2021$ by author(s) and Scientific Research Publishing Inc. This work is licensed under the Creative Commons Attribution International License (CC BY 4.0).

http://creativecommons.org/licenses/by/4.0/

\begin{abstract}
An 85-year-old female patient visited our emergency department with a history of lower abdominal pain and vomiting. Abdominal plain CT showed small bowel obstruction caused by a gallstone measuring $3 \mathrm{~cm}$ in size in the terminal ileum, gas in the gallbladder and adhesion in the gallbladder and duodenum. Thus, gallstone ileus with a cholecystoduodenal fistula was diagnosed. The gallstone was removed by the lower gastrointestinal endoscopy. Because of the high-risk status of the patient surgery, such as cholecystectomy or resection of the cholecystoduodenal fistula was not performed. Upper gastrointestinal endoscopy and radiography revealed the cholecystoduodenal fistula in the anterior wall of the duodenal bulb. Upper gastrointestinal radiography showed that the cholecystoduodenal fistula had been closed spontaneously without any complications on the 13th hospital day. Endoscopic removal of gallstone causing ileus is safe and effective as a less invasive alternative compared with surgery, although it requires condition that endoscope could reach the gallstone. The treatment method for the cholecystoduodenal fistula should be selected keeping in mind that conservative treatment, without surgery, may be effective.
\end{abstract}

\section{Keywords}

Endoscopic Removal, Small Bowel Obstruction, Cholecystoduodenal Fistula

\section{Introduction}

Gallstone ileus is a relatively rare disease that occurs when stones that were present in the gallbladder or bile duct migrate into the gastrointestinal (GI) tract via an internal bile fistula and becomes impacted in the intestinal tract which was first described by Dr Erasmus Bartholin, a Danish physician, in 1654. This 
mechanical obstruction due to impaction generally affects elderly female patients and it is difficult to select a treatment policy due to their general health condition and various pre-existing co-morbidities. The presence of a multitude of co-morbid conditions contributes to the morbidity and mortality rates associated with this condition. The main goal of treatment is the relief of intestinal obstruction by extraction of the gallstone using surgical procedures which is the traditional method of treatment. However, the surgical mortality rate is high and a less invasive approach, such as endoscopic method would be preferable.

This case report describes a case where the patient suffered gallstone ileus impacted in the terminal ileum that was successfully treated without surgery. The impacted stone was removed trans-anally using a lower gastrointestinal endoscope, while the cholecystoduodenal fistula was allowed to heal by itself.

This case report does not require the consent of the Institutional Review Board, and the patient's personal information has been anonymized. The patient has agreed for her data to be used in this paper.

\section{Case Presentation}

Patient is an 85-year-old female. Lower abdominal pain and vomiting from the previous day were noted, and the symptoms did not improve, hence she was transferred to our hospital for emergency treatment. She suffers from diabetic nephropathy, cerebral infarction, and gallbladder stone disease.

The status at the time of admission: Blood pressure 145/84 $\mathrm{mmHg}$, pulse rate 100 times/min, body temperature $36.0^{\circ} \mathrm{C}$, respiratory rate 25 times $/ \mathrm{min}, \mathrm{SpO}_{2}$ $96 \%$ (room air), and clear consciousness. The abdomen was slightly distended but soft, and tenderness was observed in the lower abdomen, while no peritoneal irritation was observed.

Blood test findings: Some inflammatory responses, renal dysfunction, and increases in hepatobiliary system enzymes were observed (Table 1).

Table 1. Laboratory data on admission.

\begin{tabular}{cccc}
\hline \multicolumn{2}{c}{ Blood Cell Counts } & \multicolumn{2}{c}{ Biochemical Tests } \\
\hline $\mathrm{WBC}$ & $10.8 \times 10^{3} / \mu \mathrm{L}$ & $\mathrm{TP}$ & $7.3 \mathrm{~g} / \mathrm{dL}$ \\
$\mathrm{RBC}$ & $520 \times 10^{4} / \mu \mathrm{L}$ & $\mathrm{BUN}$ & $34 \mathrm{mg} / \mathrm{dL}$ \\
$\mathrm{Hb}$ & $12.3 \mathrm{~g} / \mathrm{dL}$ & Creatinine & $1.97 \mathrm{mg} / \mathrm{dL}$ \\
$\mathrm{Ht}$ & $38.9 \%$ & AST & $66 \mathrm{IU} / \mathrm{L}$ \\
$\mathrm{Plt}$ & $21.5 \times 10^{4} / \mu \mathrm{L}$ & $\mathrm{ALT}$ & $53 \mathrm{IU} / \mathrm{L}$ \\
& & Al-P & $506 \mathrm{IU} / \mathrm{L}$ \\
& & $\gamma-\mathrm{GTP}$ & $221 \mathrm{IU} / \mathrm{L}$ \\
& & LDH & $156 \mathrm{IU} / \mathrm{L}$ \\
& & $\mathrm{t}-\mathrm{Bil}$ & $0.8 \mathrm{mg} / \mathrm{dL}$ \\
& & $\mathrm{CRP}$ & $4.7 \mathrm{mg} / \mathrm{dL}$ \\
\hline
\end{tabular}


Abdominal CT examination: The gallbladder showed diffused wall thickening and intra-gallbladder emphysema, and the surrounding visceral fat concentration was elevated. The gallbladder and duodenum were close to each other (Figure 1(a)). A stone shadow of $3 \mathrm{~cm}$ in diameter with marginal calcification was found in the terminal ileum. Dilation of the small intestine on the oral side of the stone was observed (Figure 1(b)).

Based on the above findings, gallstone ileus with cholecystoduodenal fistula was diagnosed. The patient was prepared for gallstone removal using a lower gastrointestinal endoscope.

Lower gastrointestinal endoscopy: A colonoscope (PCF-H290I, manufactured by Olympus) was used. It was inserted into the terminal ileum through the anus, and the black-green stone was seen (Figure 2(a)). The stone was grasped using basket forceps (BML-V232QR-30, manufactured by Olympus) (Figure 2(b)). The stone was removed without crushing it. An ulcer, thought to be caused by mechanical irritation of the stone on the mucosa was observed, however, no perforation or necrosis was visible (Figure 2(c)).

In-hospital clinical course: The upper gastrointestinal endoscopy under the fluoroscopy on the 5th hospital day revealed a depressed lesion in the anterior wall of the duodenal bulb (Figure 3), and selective upper gastrointestinal radiography revealed cholecystoduodenal fistula (Figure 4). On the 13th hospital day, the upper gastrointestinal radiography using a gastric tube confirmed the closure of the cholecystoduodenal fistula (Figure 5). The patient was discharged on the 16th hospital day and transferred to another hospital for rehabilitation.

\section{Discussion}

Gallstone ileus is a rare disease in which gallstones have migrated into the digestive tract and obstruct the intestinal tract. It accounts for $1 \%$ to $3 \%$ of mechanical obstructions in the small intestine [1]. However, in about $25 \%$ of patients older than 65 years, gallstones are responsible for all small bowel obstruction [1]. Moreover, about $50 \%$ of the patients have a history of gallbladder disease [2]. Our patient had previously been known to have gallstones in the gallbladder,

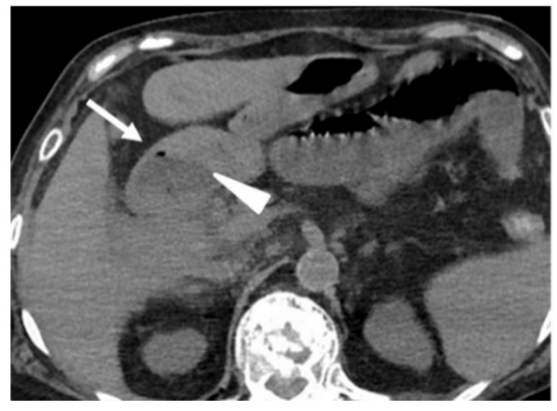

(a)

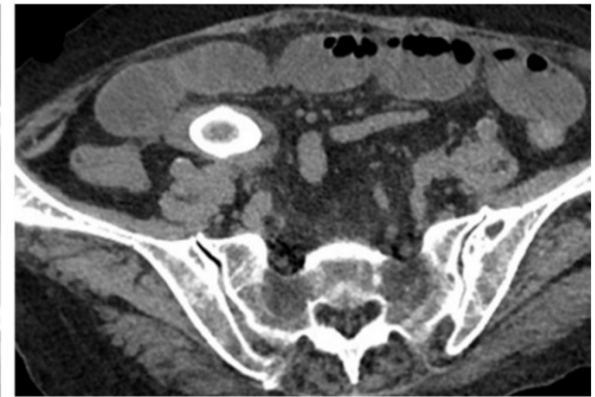

(b)

Figure 1. CT images on admission. (a) Gas (arrow) in the gallbladder and adhesion (arrowhead) between gallbladder and duodenum were observed; (b) A $3 \mathrm{~cm}$ calcified stone in the terminal ileum and dilatation of the oral side of the small intestine was observed. 


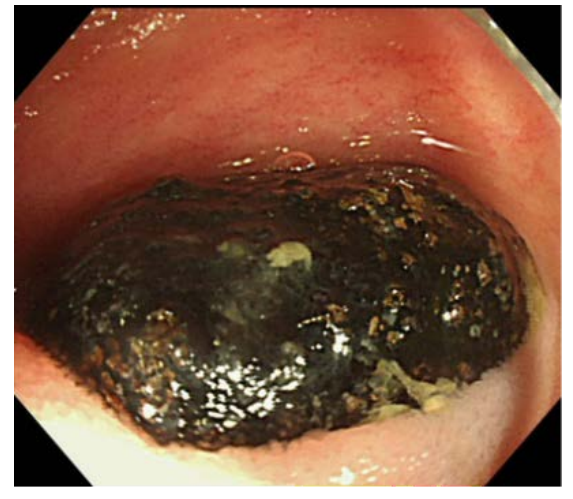

(a)

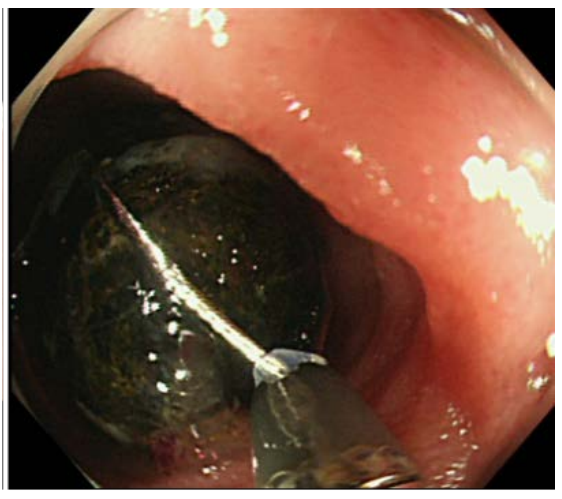

(b)

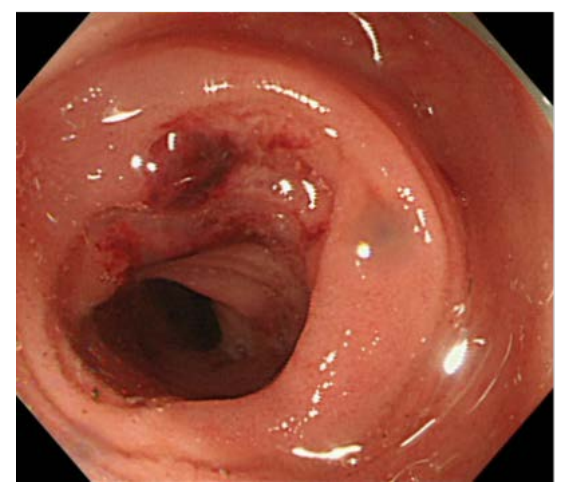

(c)

Figure 2. Findings in the terminal ileum by lower gastrointestinal endoscopy. (a) The gallstone was impacted in the terminal ileum; (b) The gallstone was grasped by forceps for endoscopic mechanical lithotomy; (c) Superficial ulcers were observed in the mucosa of terminal ileum with the impacted gallstone.

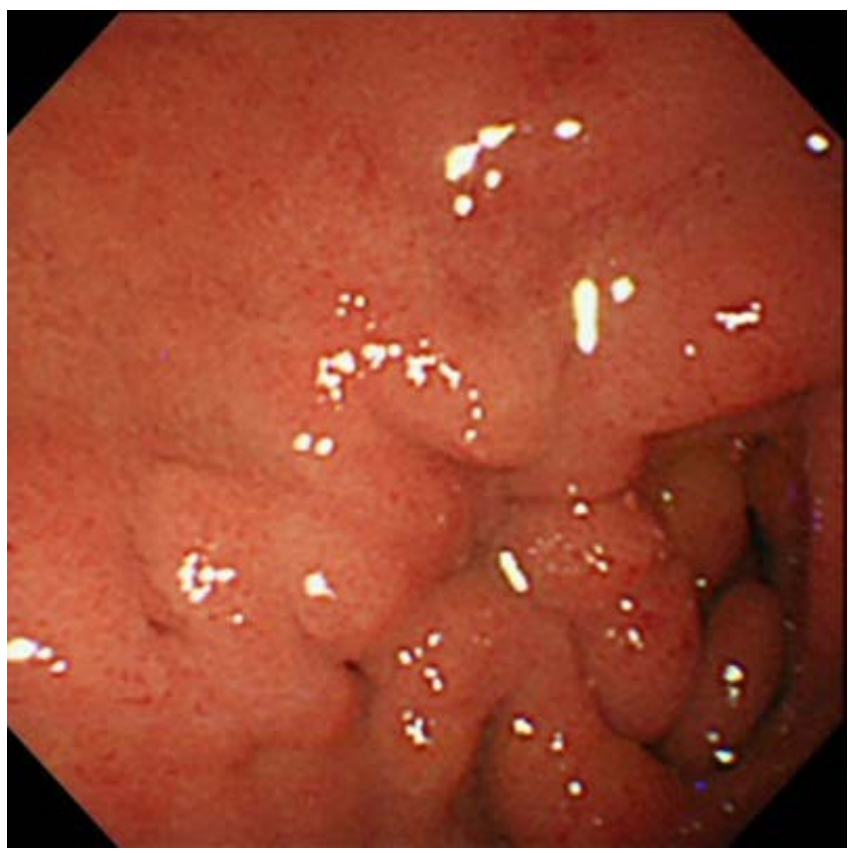

Figure 3. Findings in the duodenum by upper gastrointestinal endoscopy. An ulcerative lesion was observed on the anterior wall of the duodenal bulb. 


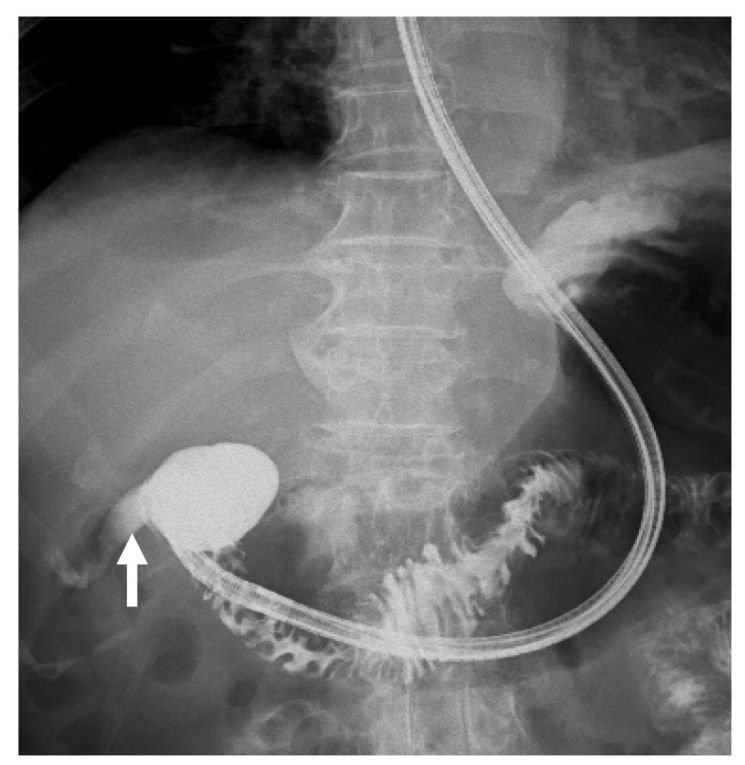

Figure 4. The image of upper gastrointestinal tract by endoscopic radiography. A cholecystoduodenal fistula (arrow) was detected on the duodenal bulb.

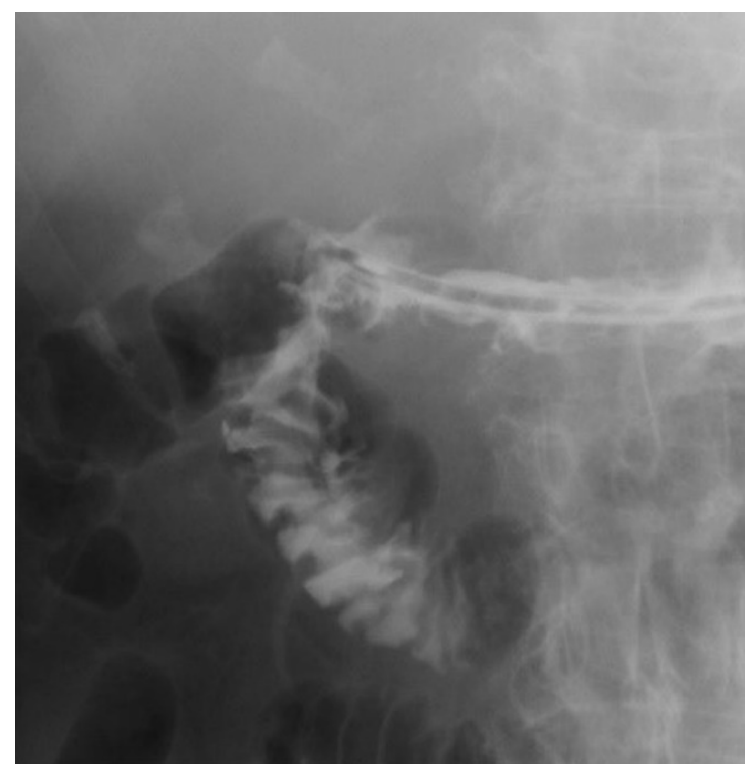

Figure 5. The image of upper gastrointestinal radiography using a gastric tube on the 13th hospital day. A closure of the cholecystoduodenal fistula was observed.

however, at the time of visit to the Emergency Centre, the abdominal CT scan showed the absence of stone in the gallbladder.

Cholecystoduodenal fistula is a complication of gallstones that causes bowel obstruction, cholangitis, weight loss, and other nonspecific symptomatology. Gallstones sometimes enter the intestinal tract through a fistula formed between the gallbladder and duodenum, stomach, or colon [3]. Cholecystoduodenal fistula is the most frequent form, found in approximately $83 \%$ of patients with gallstone ileus, while other fistulas are rare [4]. In our patient, upper gastrointestinal endoscopy and contrast examination confirmed a cholecystoduodenal fis- 
tula. This shows that the stone from the gallbladder had migrated down into the small intestine through this fistula and remained at the terminal ileum, to cause gallstone ileus. The ileum is the most common site of gallstone impaction, followed by the jejunum, and the duodenum [5]. The distal part of the ileum is the narrowest part of the intestinal tract and is supposed to have a weak propulsive activity [6]. Nakao et al. reported that the sizes of impacted gallstones ranged from $2 \mathrm{~cm}$ to $10 \mathrm{~cm}$ with a mean about $4.3 \mathrm{~cm}$ according to the analysis of 176 cases of gallstone ileus [7]. Syme et al. reported that a gallstone was usually more than $2.5 \mathrm{~cm}$ in size to cause intestinal obstruction in the normal small intestine [8]. In our patient, the stone about $3 \mathrm{~cm}$ in size was impacted at the end of the ileum, and it was judged that spontaneous stone removal from the GI tract through the anus could not be achieved. In other words, it was necessary to physically remove the obstruction from the GI tract.

Surgical management of gallstone ileus consists of 2 methods: 1) Enterolithotomy to remove the stone obstruction and 2) a second stage procedure namely biliary tract surgery (cholecystectomy with internal bile fistula repair). However, gallstone removal is usually prioritized. In actual practice, it is common to start with conservative treatment in anticipation of spontaneous excretion of the stone from the GI tract. Nonetheless, once gallstone ileus occurs, only about $1.3 \%$ of the patients passed stones spontaneously through the anus [2].

In many cases, stone removal by surgery is unavoidable. Regarding surgical management, it is still controversial whether the surgical treatment of gallstone ileus with cholecystectomy and the closure of internal bile fistula should be performed in a single or separate surgery [9]. Although one-stage surgery is desirable in terms of invasive surgery, it has been reported that the mortality rate and complication rate were higher than two-stage surgical procedures [10]. In many cases, surgical treatment under general anesthesia is at high risk due to old age and various co-morbidities. For such cases, the extracorporeal shock wave lithotripsy (ESWL) or the electrohydraulic lithotripsy (EHL) may be suitable [11] [12]. Both methods are less invasive than surgery.

While there are several reports that gallstones impacted in the intestine were removed with the endoscopy, in all cases, some kind of crushing process had been performed before picking-up the stone [12] [13] [14]. In our case, because the size of the impacted gallstone was about $3 \mathrm{~cm}$, endoscopic removal of the stone was successfully performed using the basket type forceps with a diameter of $3 \mathrm{~cm}$. The gallstone was grabbed by the forceps basket, passed through the ileocecal valve with a firm grip, and the trans-anal removal was complete with the extraction of the stone. We successfully removed the stone with the lower gastrointestinal endoscopy without lithotripsy. In cases where the gallstones are visible directly through an endoscope, it may be worth trying an endoscopic removal.

When gallstone ileus surgery is performed alone, the fistula between the gallbladder and the duodenum in most cases closes in the space of a few years and would seldom appear to give rise to serious cholangitis or liver damage [15]. 
It may be an appropriate choice to limit the surgical treatment of gallstone ileus and follow up carefully afterwards. The fistula in the duodenal bulb can be easily observed over time by gastrointestinal contrast examination or endoscopy. In our case, the closure of the fistula was confirmed by upper gastrointestinal contrast examination. Cholecystoduodenal fistula may be expected to close spontaneously, and the treatment policy should be decided case by case depending on the clinical status of the patient.

\section{Conclusion}

This case of gallstone ileus in which the gallstone impacted at the terminal ileum was successfully treated without performing any abdominal surgery. The stone was removed endoscopically, and the gallbladder duodenal fistula was allowed to heal by itself. In a selected group of patients, conservative treatment that uses a low invasive approach using the endoscopic method may be a better curative therapy.

\section{Conflicts of Interest}

The authors declare no conflicts of interest regarding the publication of this paper.

\section{References}

[1] Kirchmayr, W., Mühlmann, G., Zitt, M., Bodner, J., Weiss, H. and Klaus, A. (2005) Gallstone Ileus: Rare and Still Controversial. ANZ Journal of Surgery, 75, 234-238. https://doi.org/10.1111/j.1445-2197.2005.03368.x

[2] Reisner, R.M. and Cohen, J.R. (1994) Gallstone Ileus: A Review of 1001 Reported Cases. American Surgeon, 60, 441-446.

[3] Rodriguez-Sanjuan, J.C., Casado, F., Fernandez, M.J., Morales, D.J. and Naranjoet, A. (1997) Cholecystectomy and Fistula Closure versus Enterolithotomy Alone in Gallstone Ileus. BJS (British Journal of Surgery), 84, 634-637. https://doi.org/10.1046/j.1365-2168.1997.02637.x

[4] Freitag, M., Elsner I, Günl, U., Albert, W. and Ludwig, K. (1998) Clinical and Imaging Aspects of Gallstone Ileus. Experiences with 108 Individual Observations. Der Chirurg, 69, 265-269. https://doi.org/10.1007/s001040050410

[5] Doko, M., Zovak, M., Kopljar, M., Glavan, E., Ljubicic, N. and Hochstädter, H. (2003) Comparison of Surgical Treatments of Gallstone Ileus: Preliminary Report. World Journal of Surgery, 27, 400-404. https://doi.org/10.1007/s00268-002-6569-0

[6] Svartholm, E., Andrén-Sandberg, A., Evander, A., Järhult, J. and Thulin, A. (1982) Diagnosis and Treatment of Gallstone Ileus. Report of 83 Cases. Acta Chirurgica Scandinavica, 148, 435-438.

[7] Nakao, A., Okamoto, Y., Sunami, M., Fujita, T. and Tsuji, T. (2008) The Oldest Patient with Gallstone Ileus: Report of a Case and Review of 176 Cases in Japan. The Kurume Medical Journal, 55, 29-33. https://doi.org/10.2739/kurumemedj.55.29

[8] Syme, R.G. (1989) Management of Gallstone Ileus. Canadian Journal of Surgery, 32, 61-64.

[9] Halabi, W.J., Kang, C.Y., Ketana, N., Lafaro, K.J., Nguyen, V.Q., Stamos, M.J., Imagawa, D.K. and Demirjian, A.N. (2014) Surgery for Gallstone Ileus: A Nationwide 
Comparison of Trends and Outcomes. Annals of Surgery, 259, 329-335.

https://doi.org/10.1097/SLA.0b013e31827eefed

[10] Theodoros, E.P., Konstantinos, S.A., Basilios, T.P., Pavlidis, T.E., Atmatzidis, K.S., Papaziogas, B.T. and Papaziogas, T.B. (2003) Management of Gallstone Ileus. Journal of Hepato-Biliary-Pancreatic Surgery, 10, 299-302.

https://doi.org/10.1007/s00534-002-0806-7

[11] Meyenberger, C., Michel, C., Metzger, U. and Koelz, H.R. (1996) Gallstone Ileus Treated by Extracorporeal Shockwave Lithotripsy. Gastrointestinal Endoscopy, 43, 508-511. https://doi.org/10.1016/S0016-5107(96)70297-3

[12] Zielinski, M.D., Ferreira, L.E. and Baron, T.H. (2010) Successful Endoscopic Treatment of Colonic Gallstone Ileus Using Electrohydraulic Lithotripsy. World Journal of Gastroenterology, 16, 1533-1536. https://doi.org/10.3748/wjg.v16.i12.1533

[13] Lübbers, H., Mahlke, R. and Lankisch, P.G. (1999) Gallstone Ileus: Endoscopic Removal of a Gallstone Obstructing the Upper Jejunum. Journal of Internal Medicine, 246, 593-597. https://doi.org/10.1046/j.1365-2796.1999.00597.x

[14] Moriai, T., Hasegawa, T., Fuzita, M., Kimura, A., Tani, T. and Makino, I. (1991) Successful Removal of Massive Intragastric Gallstones by Endoscopic Electrohydraulic Lithotripsy and Mechanical Lithotripsy. American Journal of Gastroenterology, 86, 627-629.

[15] Räf, L. and Spangen, L. (1971) Gallstone Ileus. Acta Chirurgica Scandinavica, 137, 665-675. 